Supplement of Biogeosciences, 17, 47-54, 2020 https://doi.org/10.5194/bg-17-47-2020-supplement (C) Author(s) 2020. This work is distributed under the Creative Commons Attribution 4.0 License.

(c) (1)

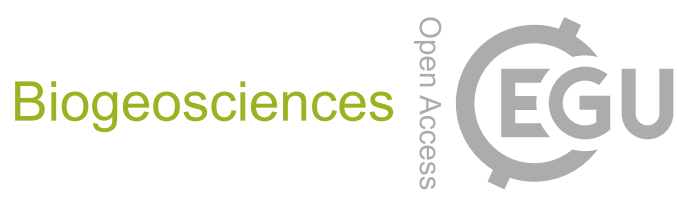

Supplement of

\title{
The capacity of northern peatlands for long-term carbon sequestration
}

Georgii A. Alexandrov et al.

Correspondence to: Victor A. Brovkin (victor.brovkin@mpimet.mpg.de)

The copyright of individual parts of the supplement might differ from the CC BY 4.0 License. 


\section{S1 Methods}

\section{S1.1 Model equations}

The height of steady-state water table above the level of draining streams, $h$, satisfies the equation:

$$
\left\{\begin{array}{c}
\frac{\partial}{\partial x}\left(K h \frac{\partial h}{\partial x}\right)+U=0 \\
h(-a)=h(a)=0
\end{array}\right.
$$

where $K$ is hydraulic conductivity, $U$ is effective rainfall, the part of precipitation that percolates into drainage streams as groundwater runoff, and $a$ is the half-distance between the drainage streams.

This equation has the solution:

$$
\left\{\begin{array}{c}
h=\sqrt{\frac{U}{K}\left(a^{2}-x^{2}\right)} \\
x \leq a
\end{array}\right.
$$

suggesting that the maximum height of water table, which is reached at the drainage divide, that is at $x=0$, is equal to

$$
h_{\max }=a \sqrt{U / K}
$$

In the case of a flat ground surface, the maximum depth of peat layer that occurs at the drainage divide could not exceed $\left(h_{\text {max }}-g(0)+d\right)$ meters, where $g$ is the height of mineral ground surface and $d$ is the maximum depth of acrotelm.

The empirical relationship between perennial streams density, $\mathrm{D}_{\mathrm{P}}$, and PET/P (Wang \& Wu, 2013):

$$
D_{P}=0.444 /(P E T / P)(\mathrm{S} 4)
$$

allows us to make an assumption about the geographic distribution of one of the model parameters related to landscape morphology. Assuming that $a=1 /\left(2 \mathrm{D}_{\mathrm{P}}\right)$, we come to an equation that links $a$ and PET/P:

$a=\gamma \operatorname{PET} / P$

where $\gamma=1.1 \mathrm{~km}$. This turns equation (S3) to

$h_{\max }=\gamma \frac{P E T}{P} \sqrt{U / K}$

and allows us to calculate $h_{\max }$ based on climatological data.

The point of $h$ and $g$ intersection, $\mathrm{x}_{0}$, is determined by the equation

$h\left(x_{0}\right)=g$

that gives

$$
x_{0}=\sqrt{a^{2}-g^{2} K / U}
$$

Then, assuming some symmetry in sense of distance to draining streams, one may estimate the fraction of the watershed area where the water table is close to the ground surface as the ratio of $\mathrm{x}_{0}^{2}$ to $\mathrm{a}^{2}$ :

$f_{P}=1-\frac{g^{2}}{a^{2}} K / U$ 
and taking into account equation (S5) come to

$f_{P}=1-\frac{g^{2}}{\gamma^{2}} \frac{K}{U}\left(\frac{P}{P E T}\right)^{2}$

Taking spatial prediction of the histic soil probability, provided by the soil information system SoilGrids, as an “observed" value of $f_{P}$ makes it possible to estimate $K$ :

$$
K=\left(1-f_{P, o b s}\right) U \frac{\gamma^{2}}{g^{2}}\left(\frac{P E T}{P}\right)^{2}
$$

Substituting $K$ given by equation (S11) to equation (S6) gives

$$
h_{\max }=\frac{g}{\sqrt{1-f_{P, o b s}}}
$$

and the following equation for the maximum depth of peat that could be accumulated above the average height of the ground surface:

$$
p_{\max }=h_{\max }-g+d=g \frac{1-\sqrt{1-f_{P, o b s}}}{\sqrt{1-f_{P, o b s}}}+d
$$

Assuming some similarity in sense of the shape of peat strata, one may calculate the area of a strata as a function of its height

$$
F(z)=b\left(a^{2}-z^{2} K / U\right)
$$

where $b$ is a coefficient of proportionality, which is equal to $\pi$ in the case of disc-shaped strata, and calculate the volume of peat mound as

$$
V=\int_{g}^{h_{\max }} b\left(a^{2}-z^{2} K / U\right) d z=b a^{2}\left(h_{\max }-g\right)-b \frac{1}{3}\left(h_{\max }^{3}-g^{3}\right)(K / U)
$$

Dividing V by $b a^{2}$ (an approximation of the watershed area) and taking into account equation (S3) gives

$$
V /\left(b a^{2}\right)=\left(h_{\max }-g\right)-\frac{1}{3}\left(h_{\max }-g\left(\frac{g}{h_{\max }}\right)^{2}\right)
$$

and therefore the maximum carbon stock in a grid cell where peatlands occupy $f_{P, o b s}$ fraction of area can be estimated using the following equation:

$$
p_{C, \max }=c \times A \times\left(\left(h_{\max }-g\right)-\frac{1}{3}\left(h_{\max }-g\left(\frac{g}{h_{\max }}\right)^{2}\right)+d \times f_{P, o b s}\right)
$$

where $h_{\max }$ is given by equation (S12), $c$ is the bulk carbon density of peat, in $\mathrm{gC} \mathrm{m}^{-3}$ and $\mathrm{A}$ is the area of the grid cell, in $m^{2}$.

The use of regular grid for representing the spatial distribution of peatland area leads to different interpretations of the data of the fraction of grid cell covered by histosols. For example, if $36 \%$ of a grid cell is covered by histosols, one may assume that peatlands cover $36 \%$ of each watershed within this grid cell (a uniform interpretation), or that only $48 \%$ of watersheds are occupied by peatlands, and they cover $75 \%(0.48 \times 75=36)$ of each of these watersheds (a clumped interpretation).

The variety of possible interpretations could be parameterized using the following equation: 
where $f_{W P}$ is the fraction of cell area covered by watersheds occupied by peatlands, and $f_{P W}$ is the fraction of area they cover at each of these watersheds.

Considering these interpretations leads to the generalization of the Equation (S17):

$$
\begin{aligned}
& p_{C, \max }=c \times f_{W P} \times A \times\left(\left(h_{\max }-g\right)-\frac{1}{3}\left(h_{\max }-g\left(\frac{g}{h_{\max }}\right)^{2}\right)+d \times f_{P W}\right)= \\
& =c \times A \times\left(\left[\left(h_{\max }-g\right)-\frac{1}{3}\left(h_{\max }-g\left(\frac{g}{h_{\max }}\right)^{2}\right)\right] \times f_{W P}+d \times f_{P W} \times f_{W P}\right)
\end{aligned}
$$

that, based on the Equation (S18), could be written as follows

$$
\left\{\begin{array}{c}
p_{C, \text { max }}=c \times A \times\left(\left(h_{\max }-g\right)-\frac{1}{3}\left(h_{\max }-g\left(\frac{g}{h_{\max }}\right)^{2}\right) \frac{f_{P, o b s}}{f_{P W}}+d \times f_{P, o b s}\right) \\
h_{\max }=\frac{g}{\sqrt{1-f_{P W}}}
\end{array}\right.
$$

The value of the hydraulic conductivity coefficient, equation (S11) could be used as an indicator of clustered peatland distribution within a grid cell. If it is above the typical value, $K_{c}$, then one may assume that $f_{P W}$ should be set at the value

$f_{P W}=1-\frac{g^{2}}{\gamma^{2}} \frac{K_{c}}{U}\left(\frac{P}{P E T}\right)^{2}$

that brings $K$ to the value typical for peat.

\section{S1.2 Data processing}

\section{S1.2.1 Depth to bedrock (displayed at the Figure 1 of the manuscript)}

The file, BDTICM_M_10km_ll.tif, containing the data on the depth to bedrock was downloaded from the following FTP:

\section{ftp://ftp.soilgrids.org/data/aggregated/10km/}

and converted into ASCII format using the utility program distributed with GDAL ${ }^{1}$ :

\$ gdal_translate -of AAIGrid BDTICM_M_10km_1l.tif BDTICM_M_10km_ll.asc

The obtained file, BDTICM_M_10km_ll.asc, consists of 1400 lines. Each line contains 3600 integers representing the

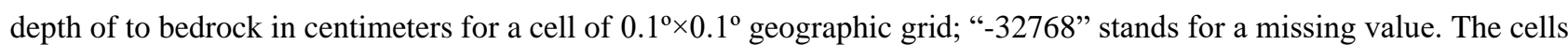
fall within a rectangle. The coordinates of its corners are $84 \mathrm{~N}$ and $180 \mathrm{~W}$ (upper left), $56 \mathrm{~S}$ and $180 \mathrm{~W}$ (lower left), $84 \mathrm{~N}$ and $180 \mathrm{E}$ (upper right), 56S and 180E (lower right).

Then, 60 lines containing only missing values (-32768) were added to the beginning of the BDTICM_M_10km_1l.asc to obtain the file, "bdt.asc", that was used in further calculations. This file contains 1460 lines. The coordinates of the upper left corner of the grid rectangle represented by the data in this file are $90 \mathrm{~N}$ and $180 \mathrm{~W}$.

\footnotetext{
${ }^{1}$ http://www.gdal.org/index.html
} 
The content of this file is displayed at the Figure 1 of the manuscript using MapWindow 4.8., a free spatial data viewer and geographic information system (https://www.mapwindow.org/).

\section{S1.2.2 The fraction of histosols (displayed at the Figure 2 of the manuscript)}

The original file, histosol_frac_0.1_deg.nc, containing the data on the fraction of each $0.1^{\circ} \times 0.1^{\circ}$ grid cell covered by soils of histosol type was converted to ASCII format using utility provided by Mathematica:

data = Import["histosol_frac_0.1_deg.nc", \{"Datasets", "histosol_frac"\}];

Export["histosol_frac_0.1_deg.dat", data, "Table", "FieldSeparatōs" -> " "]

Then fractions were changed to percentages and the order of lines were reverted: the coordinates of the upper left corner of the grid rectangle represented by the data in the file 'histosol_frac_int.asc' are 90N and 180W.

The content of this file is displayed at the Figure 2 of the manuscript using MapWindow 4.8.

\section{S1.2.3 The conductivity-dependent estimate of the potential peat carbon stock in a grid cell (displayed at the Figure 3 of the manuscript)}

The conductivity-dependent estimate of the potential carbon stock in a grid cell is calculated using the following algorithm:

1. calculate $\mathrm{K}$, equation ( $\mathrm{S} 11$ ), based on the data on the depth to bedrock (bdt.asc), on the fraction of histosols (histosol_frac_int.asc), on the annual precipitation (pann_gswp.asc), on the annual evapotranspiration (petann_gswp.asc), and on the mean annual temperature (tann_gswp.asc);

2. if mean annual temperature is below $-2^{\circ} \mathrm{C}$, then set $\mathrm{g}$ at the value that is equal to $2 \mathrm{~m}$;

3. if $\mathrm{K}>\mathrm{K}_{\mathrm{c}}$, then use equation ( $\left.\mathrm{S} 18\right)$ to set $f_{P W}$ value $\left(f_{P W}=f_{P, o b s}\right.$ by default, $\left.\mathrm{K}_{\mathrm{c}}=157 \mathrm{~m} \mathrm{yr}^{-1} \approx 0.5 \times 10^{-5} \mathrm{~m} \mathrm{~s}^{-1}\right)$;

4. calculate the potential peat carbon stock in a grid cell using equation (S19).

Table S1. Conductivity-dependent estimate of the total potential carbon stock in the northern peatlands as dependent on $\mathrm{K}_{\mathrm{c}}$.

\begin{tabular}{|l|l|}
\hline $\mathbf{K}_{\mathbf{c}}$ & Carbon stock, PgC \\
\hline $\mathbf{7 0}$ & 1071 \\
\hline $\mathbf{1 5 0}$ & 885 \\
\hline $\mathbf{1 5 5}$ & 878 \\
\hline $\mathbf{1 5 7}$ & 875 \\
\hline $\mathbf{1 6 0}$ & 870 \\
\hline $\mathbf{2 0 0}$ & 825 \\
\hline $\mathbf{3 0 0}$ & 765 \\
\hline $\mathbf{3 5 0}$ & 750 \\
\hline $\mathbf{4 5 0}$ & 730 \\
\hline
\end{tabular}




\section{S1.2.4 The uniform estimate of the potential peat carbon stock in a grid cell}

The uniform estimate of the potential peat carbon stock in a grid cell is calculated using equation (S19), where $f_{P W}=f_{P, o b s}$.

\section{S1.2.5 The clumped estimate of the potential peat carbon stock in a grid cell}

The clumped estimate of the potential peat carbon stock in a grid cell is calculated using equation (S19), where $f_{P W}=0.75$.

\section{S2 Empirical model of peat accumulation}

In 2011, Yu analysed available peat core data, estimated the total carbon stock in the northern peatlands at 1000-year intervals, and summarized the obtained estimates with the model of peat accumulation:

$P_{C}(t)=(\beta / \alpha)\left(1-e^{-\alpha(12000-\mathrm{t})}\right)$

where $t$ is number of years before present, $\alpha=0.0000855$ is the peat decay constant, $\beta=74 \mathrm{TgC} / \mathrm{yr}$ is the rate of carbon addition to pool of peat deposits.

This model, representing the balance between the rate of carbon gain and the rate of carbon loss, suggests that the carbon stock was negligible 12000 years ago, $P_{C}(12000)=0$ and equals to $561 \mathrm{PgC}$ at present, $P_{C}(0)=561$. It also suggests that the carbon stock may reach $741 \mathrm{PgC}$ within next 10000 years, $P_{C}(-10000)=741,818 \mathrm{PgC}$ within next 20000 years, $P_{C}(-$ $10000)=818$, but may not exceed $875 \mathrm{PgC}(\beta / \alpha=875 \mathrm{PgC})$. The latter value is the estimate of the potential carbon stock in northern peatlands implied by Yu's model of peat accumulation.

The accuracy of this estimate could be derived from the range of uncertainty for the present value of the carbon stock, from 473 to $621 \mathrm{PgC}$, reported in Yu's paper. The lower values could be reproduced with Equation (S19) either through decreasing $\beta$ by $15.6 \%$ or through increasing $\alpha$ by $41.7 \%$. Decreasing $\beta$ by $15.6 \%$ reduces the estimate of potential carbon stock by $15.6 \%$, whereas increasing $\alpha$ by $41.7 \%$ reduces it by $29.5 \%$. Hence, assuming that the present carbon stock could be as low as $473 \mathrm{PgC}$ leads to assumption that the potential carbon stock could be as low as $618 \mathrm{PgC}$.

Similarly, assuming that the present carbon stock could be as low as $621 \mathrm{PgC}$ leads to assumption that the potential carbon stock could be as high as $1140 \mathrm{PgC}$. The high estimate of the present carbon stock could be reproduced with Equation (S19) either through increasing $\beta$ by $10.7 \%$ or through decreasing $\alpha$ by $23.3 \%$. Increasing $\beta$ by $10.7 \%$ raises the estimate of potential carbon stock by $10.7 \%$, whereas decreasing $\alpha$ by $23.3 \%$ raises it by $30.3 \%$.

Attributing the uncertainty in the estimate of the present carbon stock to the uncertainty in the estimates of $\alpha$ and $\beta$ allows us to evaluate the uncertainty in the estimate of the potential carbon sink implied by Equation (S19). The values of $P_{C}(0)$ ranges from 467 to $671 \mathrm{PgC}$ for $\alpha=0.0000875 \pm 0.000006$ and $\beta=0.0748 \pm 0.0052$, and if $\alpha$ and $\beta$ fall within these intervals, then values of $\beta / \alpha$ ranges from 760 to $1006 \mathrm{PgC}$.

\section{S3 A comparison between the potential and observed peat carbon density}

Potential peat carbon density $\left(p_{\mathrm{c}, \mathrm{d}}\right)$ at the central part of peatland estimated is calculated as follows:

$$
\left\{\begin{array}{c}
p_{c, d}=c \times\left(h_{\max }-\mathrm{g}+d\right) \\
h_{\max }=\frac{g}{\sqrt{1-f_{P, o b s}}}
\end{array}\right.
$$

where $f_{\mathrm{p}, \mathrm{obs}}$ is set at 0.75 in case clumped estimate, and in case of uniform estimate its value is retrieved from the histosol_frac_int.asc file; $g$ is retrieved from bdt.asc file. 


\section{References}

Wang, D. \& Wu, L.: Similarity of climate control on base flow and perennial stream density in the Budyko framework, Hydrol. Earth Syst. Sci., 17, 315-324, 2013

$\mathrm{Yu}, \mathrm{Z}$.: Holocene carbon flux histories of the world's peatlands: Global carbon-cycle implications, The Holocene, 21(5), 761-774, doi:10.1177/0959683610386982, 2011. 